\title{
Thermal and collisional corrections to the ordinary wave
}

\author{
By F. N. ARUMI AND M. E. OAKES \\ Department of Physics, University of Texas at Austin
}

(Received 5 March 1971)

A moment approach is used to find the thermal and collisional corrections to the ordinary wave in a plasma. Results are given that include like- and unlikeparticle collisions.

\section{Introduction}

Electromagnetic waves propagating perpendicular to a static magnetic field $B_{0}$ can be distinguished according to their polarization. Waves, whose electric field lies along the static magnetic field, are labelled ordinary; the extraordinary wave is elliptically polarized in the plane perpendicular to $B_{0}$. These waves have been studied by many authors. Excellent surveys are found in Stix (1962) and Ginsberg (1964). More recently (Hamasaki 1968; Bornatici \& Lee 1970; Lee \& Armstrong 1971), interest has developed in the stability of the ordinary wave.

A cold-plasma treatment of the ordinary wave predicts phase velocities independent of $B_{0}$; similarly, a fluid description utilizing a scalar pressure also indicates no magnetic field dependence. A kinetic treatment based on the Vlasov equation yields magnetic field and thermal corrections to this mode (Stix 1962; Dnestrovskii \& Kostomarov 1961). In this paper, we show that a fluid description is possible; this approach, in addition to providing insight into the physical nature of the correction terms, also easily permits the inclusion of like and unlike collisions. The collisionless results obtained are in full agreement with those found by the kinetic approach. It is important to note that these corrections cannot be found by using the pressure tensor given by Chapman \& Cowling (1960), or using the Chew-Goldberger-Low equations as modified by Macmahon (1965).

\section{Theory and discussion}

The time development of the one-body distribution function is given by

$$
\frac{\partial f(1)}{\partial t}+\mathbf{v} \cdot \frac{\partial f(1)}{\partial \mathbf{r}}+\frac{q}{m}\left(\mathbf{E}+\frac{1}{c} \mathbf{v} \times \mathbf{B}\right) \cdot \frac{\partial f(1)}{\partial \mathbf{v}}=K(2),
$$

where $f(1)$ is the one-body distribution function, $v$ the microscopic particle velocity, $q$ the electric charge, $m$ the particle mass, $\mathbf{E}$ the electric field, $\mathbf{B}$ the magnetic field, and $K(2)$ the two-body correlation term. In the following development, it is assumed that the unperturbed distribution function is Maxwellian. The perturbation to the distribution function will not be isotropic. The degree of departure 
from isotropy will be postulated by retaining terms through the third moment of the distribution (heat transfer tensor), and by neglecting the contributions from the higher moments.

The contribution of the correlation term to the moment equations have been chosen so that they will satisfy the following criteria: (i) The number of particles of each species will remain constant. (ii) The temperature of the different species will be different, i.e. the contribution of unlike particle collisions to the energy equation will be neglected. In addition, of course, these must satisfy both energy and momentum conservation in their respective moment equations. In what follows, we assume the ions and neutrals to be stationary. The linearized moment equations become

$$
\left.\begin{array}{c}
\frac{\partial N_{1}}{\partial t}+N_{0} \frac{\partial V_{i}}{\partial x_{i}}=0 \\
m N_{0} \frac{\partial V_{i}}{\partial t}+\frac{\partial p_{i j}}{\partial x_{j}}+e N_{0}\left(E_{i}+\frac{1}{c} \epsilon_{i j 3} V_{j} B_{0}\right)=-m N_{0} \gamma V_{i}, \\
\frac{\partial p_{i j}}{\partial t}+\frac{\partial Q_{i j k}}{\partial x_{k}}+p_{0}\left(\delta_{i j} \frac{\partial V_{k}}{\partial x_{k}}+\frac{\partial V_{j}}{\partial x_{i}}+\frac{\partial V_{i}}{\partial x_{j}}\right) \\
+\omega_{c}\left(\epsilon_{i r 3} p_{r j}+\epsilon_{j r 3} p_{r i}\right)=-\nu\left(p_{i j}-p \delta_{i j}\right) \\
\begin{array}{rl}
\frac{\partial Q_{i j k}}{\partial t}+\omega_{c}\left(\epsilon_{j r 3} Q_{r k i}+\epsilon_{r k 3} Q_{r j i}\right. & \left.+\epsilon_{i r 3} Q_{r j k}\right) \\
& =-\nu Q_{i j k}+p_{0} \gamma\left(\delta_{i j} V_{k}+\delta_{j k} V_{i}+\delta_{i k} V_{j}\right)
\end{array}
\end{array}\right\}
$$

Here, $e$ is the electronic charge, $\omega_{c}$ the cyclotron frequency. The quantities subscripted with a zero are the unperturbed quantities. Summation over repeated indices is implied. $\gamma$ represents the effective unlike-particle collision frequency. $\nu$ represents the like-particle collision frequency. The like-particle correlation terms were derived using the Krook's model for the correlation term.

$$
\begin{aligned}
N & \equiv \int f d^{3} v \text { is the particle density, } \\
V_{i} & =\frac{1}{N} \int v_{i} f d^{3} v \text { is the macroscopic particle velocity, } \\
p_{i j} & =m \int\left(v_{i}-V_{i}\right)\left(v_{j}-V_{j}\right) f d^{3} v \text { is the pressure tensor, }
\end{aligned}
$$

and $\quad Q_{i j k}=m \int\left(v_{i}-V_{i}\right)\left(v_{j}-V_{j}\right)\left(v_{k}-V_{k}\right) f d^{3} v$ is the heat-transfer tensor.

The uniform static magnetic field has been taken to define the 3-direction. The ordinary mode is definable for waves propagating perpendicular to the magnetic field. The electric field of this wave is parallel to $B_{0}$. The macroscopic velocities of the particles is finite only in the direction of the magnetic field. We let the direction of propagation of the wave define the 2-direction.

$$
\left.\begin{array}{c}
\frac{\partial N_{1}}{\partial t}=0 \\
m N_{0} \frac{\partial V}{\partial t}+\frac{\partial p_{23}}{\partial x_{2}}+e N_{0} E=-m N_{0} \gamma V
\end{array}\right\}
$$


The temperature correction to the ordinary mode comes through the shear term between the directions of wave propagation and the magnetic field. Thus, we should not expect to see thermal corrections to this mode when we use a model based on an adiabatic (or isothermal) equation of state. This shear term is given by the relations,

$$
\begin{gathered}
\frac{\partial p_{23}}{\partial t}+\frac{\partial Q_{223}}{\partial x_{2}}+p_{0} \frac{\partial V}{\partial x_{2}}-\omega_{c} p_{13}=-\nu p_{23} \\
\frac{\partial p_{13}}{\partial t}+\omega_{c} p_{23}=-\nu p_{13} \\
\frac{\partial Q_{223}}{\partial t}=-\nu Q_{223}+\gamma p_{0} V
\end{gathered}
$$

In obtaining (6), we have used the fact that $Q_{123}=0$. Letting the time-space dependences go as $\exp (-i \omega t)$, for wave frequency $\omega$, and $\exp \left(i k x_{2}\right)$, respectively, we obtain

$$
p_{23}=p_{0} \frac{k}{\omega} \frac{[1+i(g+\mu)] V}{\left[(1+i \mu)^{2}-y^{2}\right]},
$$

for the wave vector $k$, where $\mu \equiv \nu / \omega, g=\gamma / \omega$, and $y \equiv \omega_{c} / \omega$. Substituting (7) into the equation of motion (3), we obtain

$$
V=\frac{-i e}{m \omega(1+i g)} E+\frac{u n^{2}}{1+i g}\left[\frac{1+i(g+\mu)}{(1+i \mu)^{2}-y^{2}}\right] V,
$$

where

$$
n \equiv \frac{c k}{\omega}, \quad u=\frac{p_{0}}{m N_{0} c^{2}}
$$

$n$ the index of refraction, and $u$ a dimensionless ratio.

In order to be consistent with the formal moment expansion, (8) is solved by treating the product $u n^{2}$ as a perturbation parameter in the velocity. The zeroorder (in $u n^{2}$ ) solution is the cold-plasma solution,

$$
V_{c}=\frac{-i e}{m \omega(1+i g)} E
$$

the first temperature corrections are obtained by substituting (9) into the right side of (8):

$$
V=\frac{-i e}{m \omega(1+i g)}\left\{1+\frac{u n^{2}[1+i(g+\mu)]}{\left[(1+i \mu)^{2}-y^{2}\right](1+i g)}\right\} E \text {. }
$$

This method of solution is valid, provided the thermal contribution remains small compared to the cold-plasma solutions, i.e.

$$
1 \gg\left|\frac{u n^{2}[1+i(g+\mu)]}{\left[(1+i \mu)^{2}-y^{2}\right](1+i g)}\right| \text {. }
$$

For the special case of no collisions $(g=\mu=0),(11)$ becomes

$$
\left|u n^{2} /\left(1-y^{2}\right)\right| \ll 1 ;
$$

for large frequencies $\left(y^{2} \ll 1\right)$, this condition becomes

$$
\left|u n^{2}\right| \ll 1, \text { or } K T_{e} / m \ll v_{\text {phase }}^{2},
$$


the standard hydrodynamic condition. For small frequencies $\left(y^{2} \gg 1\right)$, the condition becomes

$$
\left|u n^{2} / y^{2}\right| \ll 1 \text { or } R^{2} k^{2} \ll 1,
$$

where $R$ is the electron Larmor radius. For no collisions, in fact, (10) can be shown to be analytically the same as the result obtained by integrating the Vlasov equation for finite Larmor radius effects. The total current density is given by

$$
\left.\begin{array}{c}
J=-e N_{0} V, \\
J=\frac{i \Omega \omega}{4 \pi(1+i g)}\left\{1+\frac{n^{2} u[1+i(g+\mu)]}{(1+i g)\left[(1+i \mu)^{2}-y^{2}\right]}\right\} E,
\end{array}\right\}
$$

where $\Omega=\omega_{p}^{2} / \omega^{2}$ (a dimensionless ratio), and $\omega_{p}^{2}=4 \pi e^{2} N_{0} / m$ is the plasma frequency.

We can use (12) in the wave equation,

$$
\left(n^{2}-1\right) E=J i(4 \pi / \omega)
$$

to obtain the following expression for the index of refraction:

$$
n^{2}=[1-\Omega /(1+i g)] / 1+\frac{\Omega u[1+i(g+\mu)]}{(1+i g)^{2}\left[(1+i \mu)^{2}-y^{2}\right]} .
$$

In the absence of collisions, (14) becomes

$$
n^{2}=(1-\Omega) /\left[1+\frac{\Omega u}{1-y^{2}}\right] \text {. }
$$

The first notable result is that the ordinary mode is no longer independent of the magnetic field. In addition to the cold-plasma cut-off $\Omega=1$, we obtain a cut-off at $y^{2}=1$, and a resonance at $y^{2}=1+\Omega u$. The ordinary mode propagates for $\Omega<1$. For $\Omega>1$, the ordinary mode has a propagation window in the range $1<y^{2}<1+\Omega u$.

\section{Parametric validity conditions}

Substituting (15) into (11), the parametric validity condition for the solution is obtained:

$$
\left|\frac{u(1-\Omega)}{1+\Omega u-y^{2}}\right| \ll 1 .
$$

Clearly, the solution is always valid at the cut-off $\Omega=1$, never valid at the resonance $y^{2}=1+\Omega u$. For $\Omega \gg 1$, the condition becomes

$$
\left|\frac{u \Omega}{1+\Omega u-y^{2}}\right| \ll 1,
$$

which states that (15) is not valid in the magnetic field range $1<y^{2}<1+2 \Omega u$. Although these corrections have a very restricted set of parameters that can satisfy their validity, they do serve as a probe to determine where the cold plasma predictions should break down and where higher temperature corrections are necessary. When collisions are included, the range of validity of these corrections can include the interesting region $\left(1<y^{2}<1+2 \Omega u\right)$. 


\section{Justification for the use of a scalar pressure}

The scalar pressure treatment of this wave does not introduce temperature effects. The scalar pressure approximation is valid when like-particle collisions are sufficiently large to make the pressure isotropic. We can regain this limit by writing an approximate expression for (14) when $\mu \gg 1$ :

$$
\begin{gathered}
n^{2}=\left[1-\frac{\Omega}{1+i g}\right]\left[1+\frac{i \Omega u}{\mu(1+i g)^{2}}\right], \\
\lim _{\mu \rightarrow \infty} n^{2}=1-\frac{\Omega}{1+i g},
\end{gathered}
$$

which is the scalar-pressure or cold-plasma result. This result is valid, according to (11), whenever

$$
\mu\left(1+g^{2}\right)^{\frac{1}{2}} \gg \Omega u,
$$

which is a criterion by which we can determine when a collisional plasma can be given a scalar-pressure treatment.

\section{Effects of the ion mass and temperature}

If the effects of the ion motion are included, (12) is modified to take the form,

$$
n^{2}=[1-\Omega(1+M)] /\left[1+\Omega u\left(\frac{M^{2} T}{1-M^{2} y^{2}}+\frac{1}{1-y^{2}}\right)\right],
$$

where

$$
M \equiv \text { (electron mass)/(ion mass), }
$$

and

$$
T=\text { (ion temperature)/(electron temperature) }
$$

This result predicts an additional cut-off at

and an additional resonance at

$$
y^{2} M^{2}=1
$$

$$
y^{2} M^{2}=1-\Omega u M^{2}(1+T)
$$

Since the quantity $\Omega u$ is of order unity or less, the product

$$
\Omega u M^{2}(1+T) \ll 1,
$$

and the ion resonance occurs practically at the same point as the cut-off; it is therefore quite justifiable to neglect the motion of ions.

\section{Kinetic results}

The ordinary mode, as obtained by integrating the Vlasov equation, can be written down from the components of the mobility tensor (Stix 1962). In the absence of a drift velocity and temperature anisotropy, we can obtain the transcendental equation,

$$
n^{2}=1-\Omega \sum_{S=-\infty}^{\infty} \frac{e^{-\lambda} I_{S}(\lambda)}{1+S y}
$$

where $\lambda=n^{2} u / y^{2}$ is the square ratio of the Larmor radius to the wavelength. Expanding (16) to keep linear terms in $\lambda$, we obtain (15). 


\section{Physical nature of thermal corrections}

Some insight into the physical nature of the thermal corrections may be obtained by neglecting like-particle collisions, so that an unperturbed solution can be found with anisotropic pressure, i.e. $p_{i j}^{(0)}=p_{\perp}\left(\delta_{i j}-\delta_{i 3} \delta_{j 3}\right)+p_{\| 1} \delta_{i 3} \delta_{j 3}$ (for unperturbed parallel electron pressure $p_{\|}$, and unperturbed perpendicular electron pressure $p_{\perp}$ ). Then the equations describing the perturbed pressure and heat transfer tensor take the form,

$$
\begin{aligned}
\frac{\partial p_{i j}}{\partial t}+\frac{\partial Q_{i j k}}{\partial x_{k}} & +p_{\perp}\left(\delta_{i j} \nabla \cdot \mathrm{V}+\frac{\delta V_{i}}{\partial x_{j}}+\frac{\partial V_{j}}{\partial x_{i}}\right)+\left(p_{0}-p_{\perp}\right)\left(\delta_{i 3} \delta_{j 3} \nabla \cdot \mathrm{V}+\delta_{i 3} \frac{\partial V_{j}}{\partial x_{3}}+\delta_{j 3} \frac{\partial V_{i}}{\partial x_{3}}\right) \\
& +\omega_{k}\left(p_{\|}-p_{\perp}\right)\left(\epsilon_{i 3 k} \delta_{j 3}+\epsilon_{j 3 k} \delta_{i 3}\right)-\omega_{c}\left(\epsilon_{i r 3} p_{r j}+\epsilon_{j r 3} p_{r i}\right)=0
\end{aligned}
$$

and

$$
\begin{aligned}
\frac{\partial Q_{i j k}}{\partial t} & +\omega_{c}\left(\epsilon_{i r 3} Q_{r j k}+\epsilon_{k r 3} Q_{r i j}+\epsilon_{j r s} Q_{r k i}\right) \\
& =\gamma\left\{p_{\perp}\left(\delta_{i j} V_{k}+\delta_{k i} V_{j}+\delta_{j k} V_{i}\right)+\left(p_{\|}-p_{\perp}\right)\left(\delta_{i 3} \delta_{j 3} V_{k}+\delta_{k 3} \delta_{i 3} V_{j}+\delta_{j 3} \delta_{k 3} V_{i}\right)\right\}
\end{aligned}
$$

where $\omega_{k}=B_{k} e /(m c)$, and $B_{k}$ is the magnetic field of the wave. For the particular case of the ordinary wave $\partial / \partial x_{i} \rightarrow \delta_{i 2} \partial / \partial x$ and $E\|\mathbf{V}\| \hat{e}_{3}$, these equations simplify to

$$
\begin{gathered}
\frac{\partial p_{23}}{\partial t}-\omega_{c} p_{13}=-\left[\frac{\partial}{\partial x}\left(p_{\perp} V-Q_{223}\right)+\omega_{1}\left(p_{\|}-p_{\perp}\right)\right] \\
\frac{\partial p_{13}}{\partial t}+\omega_{c} p_{23}=-\frac{\partial Q_{123}}{\partial x}
\end{gathered}
$$

but

$$
Q_{123}=0, \quad \text { and } \quad \frac{\partial Q_{223}}{\partial t}=\gamma p_{\perp} V
$$

Taking the time derivative of (19), we have

$$
\frac{\partial^{2} p_{23}}{\partial t^{2}}+\omega_{c}^{2} p_{23}=-\frac{\partial}{\partial x}\left(p_{\perp} \frac{\partial V}{\partial t}-\frac{\partial Q_{223}}{\partial t}\right)-\left(p_{\|}-p_{\perp}\right) \frac{\partial \omega_{1}}{\partial t}
$$

Using Faraday's law of induction, we get

$$
\frac{\partial^{2} p_{23}}{\partial t^{2}}+\omega_{c}^{2} p_{23}=-\frac{\partial}{\partial x}\left[p_{\perp}\left(\frac{\partial V}{\partial t}+\gamma V+\frac{e}{m} E\right)+p_{\|} \frac{e}{m} E\right]
$$

and remembering that, from the cold plasma,

$$
\frac{\partial V}{\partial t}+\gamma V+\frac{e}{m} E=0
$$

we get for the pressure term

$$
\frac{\partial^{2} p_{23}}{\partial t^{2}}+\omega_{c}^{2} p_{23}=-\frac{e p_{11}}{m} \frac{\partial E}{\partial x}
$$

so all the results discussed previously for isotropic pressure can be transposed to the anisotropic pressure case by setting the like particle collisions equal to 
zero and substituting $p_{0} \rightarrow p_{\|}$. In particular, if $p_{\perp}$ is set identically to zero, the dispersion relation,

$$
n^{2}=[1-\Omega /(1+i g)] / 1+\frac{\Omega u_{\sharp}}{(1+i g)\left(1-y^{2}\right)},
$$

is identically correct, except for the form of the collision term. Thus, the parallel pressure provides the thermal correction. A model of two contra-streaming beams along the magnetic field interacting with the wave's magnetic field results in a correction to the perturbed current along $\mathbf{B}_{\mathbf{0}}$. The density perturbation present in each beam cancel each other when summed, and maintain the pure electromagnetic character of the wave. After the current is averaged over a distribution of beams and the dispersion relation determined, the results found agree with (25). This procedure leads to the extended interpretation of $u_{\|}$as $\left(\theta_{\|}^{2}+v_{B}^{2}\right) / c^{2}, \theta_{\|}$the parallel thermal speed and $v_{B}$ the velocity of the counter-streaming beams. Equation (25), therefore, can be used to study the effects of unlike (species) particle collisions on the electromagnetic instability.

\section{Conclusions}

Thermal and collisional corrections to the ordinary wave are obtained from a moment approach. The collisionless results agree with those obtained from the Vlasov equation; they should be useful in determining the growth rates for associated instabilities. It is shown that, for the anisotropic pressure case, the parallel temperature is responsible for the thermal correction. A simple model of contra-streaming beams yields the same dispersion relation and the physical mechanism.

The research was supported by the National Science Foundation and the Texas Atomic Energy Research Foundation.

\section{REFERENCES}

Bornatici, M. \& Lee, K. F. 1970 Phys. Fluids, 13, 3007.

Chapman, S. \& Cowlnng, T. G. 1960 The Mathematical Theory of Non-uniform Gases. Cambridge University Press.

Dnestrovski, N. \& Kostomarov, D. P. 1961 Soviet Physics JETP, 13, 986.

Grnzburg, V. L. 1964 The Propagation of Electromagnetic Waves in Plasmas. Pergamon. HaMASAKr, S. 1968 Phys. Fluids, 11, 1173, 2724.

Lee, K. F. \& Armstrong, J. C. 1971 Phys. Rev. Lett. 26, 77.

Macmahon, A. 1965 Phys. Fluids, 8, 1840.

StIx, T. H. 1962 The Theory of Plasma Waves. McGraw-Hill. 